Wrona, T., Pan, I., Bell, R. E., Gawthorpe, R. L.,

Fossen, H., Brune, S. (2021): 3D seismic interpretation with deep learning: A brief introduction. - The Leading Edge, 40, 7, 524-532.

https://doi.org/10.1190/tle40070524.1 


\title{
3-D seismic interpretation with deep learning: a brief introduction
}

\author{
Thilo Wrona ${ }^{1,2 *}$, Indranil Pan ${ }^{3,4}$, Rebecca E. Bell ${ }^{5}$, Robert L. Gawthorpe ${ }^{1}$, Haakon Fossen ${ }^{6}$ \\ and Sascha Brune,
}

\author{
${ }^{1}$ Department of Earth Science, University of Bergen, Allégaten 41, N-5007 Bergen, Norway. \\ ${ }^{2}$ GFZ German Research Centre for Geosciences, Telegrafenberg, 14473 Potsdam, Germany. \\ ${ }^{3}$ Centre for Process Systems Engineering \& Centre for Environmental Policy, Imperial College London, UK. \\ ${ }^{4}$ The Alan Turing Institute, British Library, London, UK. \\ ${ }^{5}$ Department of Earth Science and Engineering, Imperial College, Prince Consort Road, London, SW7 2BP, UK. \\ ${ }^{6}$ Museum of Natural History, University of Bergen, Allégaten 41, N-5007 Bergen, Norway. \\ ${ }^{7}$ Institute of Geosciences, University of Potsdam, Potsdam-Golm, Germany. \\ *wrona@gfz-potsdam.de
}

\begin{abstract}
Understanding the internal structure of our planet is a fundamental goal of the Earth Sciences. As direct observations are restricted to surface outcrops and borehole cores, we rely on geophysical data to study the Earth's interior. Especially, seismic reflection data showing acoustic images of the subsurface, provide us with critical insights into sedimentary, tectonic and magmatic systems. The interpretation of these large, 2-D grids or 3-D seismic volumes is however time-consuming even for a well-trained person or team of people. Here, we demonstrate how to automate and accelerate the analysis of these increasingly large seismic datasets with machine learning. We are able to perform typical seismic interpretation tasks, such as the mapping of (1) tectonic faults, (2) salt bodies and (3) sedimentary horizons at high accuracy using deep convolutional neural networks. We share our workflows and scripts, encouraging users to apply our methods to similar problems. Our methodology is generic and flexible allowing an easy adaptation without major changes. Once trained, these models can analyze large volumes of data within seconds; opening a new exciting pathway to study the internal structure and processes shaping our planet.
\end{abstract}




\section{INTRODUCTION}

Deep learning is transforming the way we analyze large datasets in many scientific disciplines including medicine (e.g. Esteva et al., 2017), chemistry (e.g. Butler et al., 2018), and physics (e.g. Carleo \& Troyer, 2017). In Earth Science, we use large volumes of geophysical data to learn about the internal structure of

our planet. Seismic reflection data showing acoustic images of the subsurface are particularly important to our understanding of sedimentary, tectonic and magmatic systems. The emergence of 3-D seismic technology allows us to image geological structures in the subsurface over thousands of square kilometers at a resolution of a few tens of meters (Cartwright \& Huuse, 2005). While this technology provides fascinating insights into the Earth's interior, it does require increasing amounts of time, experience and expertise to analyze these large datasets (e.g. Bond et al., 2012).

In recent years, machine learning has thus been applied to numerous problems in seismic interpretation, such as: (1) salt detection (e.g., Guillen et al., 2015; Waldeland et al., 2018; Zhou et al, 2020), (2) fault detection (e.g., Zhang et al., 2014; Araya-Polo et al., 2017; Wu and Fomel, 2018; Wu et al., 2019; Mosser et al., 2020; Feng et al., 2021), (3) horizon mapping (e.g., Peters et al., 2019; Gramstad et al., 2020; Tschannen et al., 2020), and (4) seismic facies classification (e.g., Qian et al., 2018; Wrona et al., 2018; Li et al., 2019; Liu et al., 2019; Zhao, 2019). Most of these studies are built on recent advances in machine learning of multi-layered neural networks (i.e. deep learning). Deep learning describes a set of machine learning models that allow us to extract data representations (e.g. features, patterns) from raw data (LeCun et al., 2015). This allows us to learn where a particular geological feature is in the data without requiring engineering of a specific set of features (seismic attributes). In addition, we can use neural networks for unsupervised learning, where we do not pre-define the geological structures, which we would like to detect (Coléou et al., 2003; de Matos et al., 2007). 
With a few exceptions (e.g. Waldeland et al., 2018, Wu et al., 2019), many studies exploring deep learning in seismic interpretation do not publish their data, code or workflow. Given the complexity and sensitivity of these models, this makes it extremely difficult to replicate, compare and evaluate different approaches. Moreover, deep learning models are hardly used in practice, where seismic interpretations are almost exclusively performed by experts. Here, we explain: (1) how these techniques work; (2) how to apply them to your geophysical dataset; (3) which challenges can occur; and (4) which remedies are available. Deep learning describes a set of machine learning models that allow us to extract data representations (e.g. features, patterns) from the raw data (LeCun et al., 2015). Here, we train these models to perform typical seismic interpretation tasks, such as: (1) mapping faults, (2) extracting geobodies, and (3) tracing stratigraphic horizons in 3-D seismic reflection data. Our workflow, code and tutorials are available at: https://github.com/thilowrona/seismic deep learning. Deep learning can accelerate the analysis of geophysical datasets significantly, allowing us to study the structure and internal processes of the Earth in great detail.

\section{3-D SEISMIC REFLECTION DATA}

In this study, we exemplify our technique using 3-D seismic reflection data from the northern North Sea, where continental crust experienced (at least) two episodes of extension in the late Permian-Early Triassic and Middle Jurassic-Early Cretaceous (e.g., Bell et al., 2014). This led to the formation of the northern North Sea rift; a complex system consisting of hundreds to thousands of normal faults, which we can image using a newly-acquired volume of 3-D seismic reflection data. The dataset covers an area $35,410 \mathrm{~km}^{2}$, imaging the crust down to $20 \mathrm{~km}$ depth. This seismic cube was acquired with a G-Gun array consisting of 3 subarrays with a source array depth of 6-9 m; a source length of 16-18 m; an SP interval of $18.75 \mathrm{~m}$; source separation of $37.5 \mathrm{~m}$; a volume of $4550 \mathrm{in}^{3}$ and an air pressure of $2000 \mathrm{psi}$. The streamer 
consisted of 12 up to $8 \mathrm{~km}$ long cables with 636 channels each; a cable separation of $75 \mathrm{~m}$ and group spacing of $12.5 \mathrm{~m}$; depths of 7-50 m covering offsets of 150-8100 m. The data was recorded with a $2 \mathrm{~ms}$ sample interval; $9000 \mathrm{~ms}$ recording length; a low cut filter (2Hz-6db/oct) and high cut (200 Hz-370 $\mathrm{db} / \mathrm{oct})$ filter. The broadseis technology, used for recording, covers a wide range of frequencies (2.5-155 $\mathrm{Hz}$ ) providing high resolution depth imaging. The data was binned at $12.5 \times 18.75 \mathrm{~m}$ with a vertical sample rate of $4 \mathrm{~ms}$. The seismic data was processed in 90 steps including: divergence compensation; low cut filter $(1.5 \mathrm{~Hz}, 2.5 \mathrm{~Hz})$; noise attenuation (e.g. swell, direct wave); spatial anti-aliasing filter (12.5 m group interval); direct wave attenuation; source de-signature; de-spike; time-variant high cut filter; receiver motion correction and de-ghosting; FK filter; cold water and tidal statics; multiple modelling with adaptive subtraction; Tau-P mute; Radon de-multiple; far angle destriping; multiple attenuation; binning (75 m interval, 107 offset planes); acquisition hole infill; 5-D regularization; 3-D true amplitude pre-stack depth migration; residual move-out correction; Linear FL Radon; full offset stack with time-variant inner and out mute; acquisition footprint removal; crossline $\mathrm{K}$ filter; residual de-striping and dynamic Q-compensation. The seismic volume was zero-phase processed with SEG normal polarity; i.e., a positive reflection (white) corresponds to an acoustic impedance (density $\times$ velocity) increase with depth.
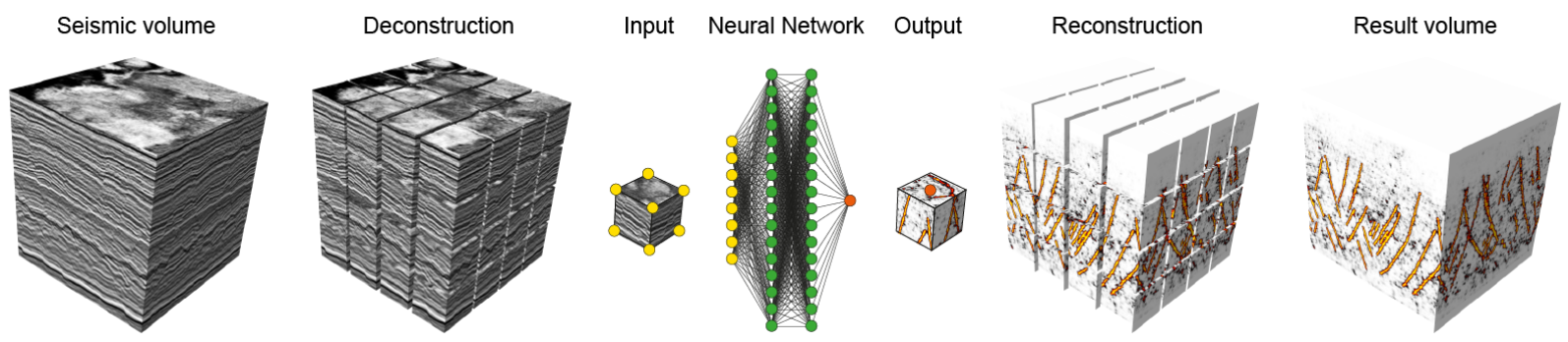

Figure 1. Schematic illustration of deep learning of 3-D seismic reflection data. We deconstruct a 3-D seismic volume into a large number of cubes, which function as input data for the neural network. Using existing seismic interpretations as labels, the neural network learns to predict the output. By predicting the output of each cube, we can reconstruct the result volume, thereby providing a full interpretation of contained fault sets and stratigraphic horizons.

\section{DEEP LEARNING}


Machine learning describes a set of algorithms and models, which learn to perform a specific task on a given data set. While classic machine learning relies on features engineered for a specific task (e.g. edges for fault detection), deep learning models derive features implicitly as part of the training. To learn these features, deep neural networks typically require a large number of examples, either labelled for supervised learning or unlabelled for unsupervised learning (e.g. LeCun et al., 2015). To generate these examples, we employ supervised learning and interpret different geological structures (faults, salt bodies and a horizon) in a subset of our data and subsequently divide this subset into a large number of patches, i.e. squares in 2-D and cubes in 3-D (Fig. 1). Next, we split all of these examples into one set for training and one for validation and one for testing. The training set is used to train the model to predict the labels. The validation set is used for an evaluation of the trained model, which allows us to fine-tune hyperparameters during training. The test set is used for an unbiased evaluation of the fine-tuned model.

Deep learning offers a variety of different types of models depending on the application. The basic building blocks of convolutional neural networks are however often the same. They typically include: (1) dense, (2) convolutional, (3) pooling, (4) batch normalisation and (5) dropout layers. A dense (or fully-connected) layer is commonly used for classification, as it connects every input (e.g. features) to every output (e.g. classes). A dense layer consists of artificial neurons, non-linear activation functions (e.g. Rectified Linear Units or ReLu, which set values below a certain threshold to a lower bound (zero), i.e. $\mathrm{f}(\mathrm{x})=\max (0, \mathrm{x})$; for more details see Nair and Hinton, 2010). These values have previously been multiplied by weights, which we fit during training. Based on the given features, this process allows dense layers to learn which class to prioritize. A convolutional layer is typically used for feature extraction converting images into activation maps highlighting where certain visual features (e.g. edges) occur in the image. A convolutional layer consists of a set of trainable filters, which generate activation maps when convolved with the input image. A key point is that these filters are flexible, as we train them to detect visual features important to the task at hand. Another important point is that convolution preserves the 
spatial structure of the image. A pooling layer is typically used for downsampling between convolutions. This layer slides a filter across the image and passes the maximum (or another measure) within the filter to the next layer. Downsampling allows us to process data at all scales irrespective of the size of the convolutional layer. Batch normalization is a technique to normalize activations (zero-mean and unit standard deviation) in intermediate layers of neural networks. Although the exact reason is still investigated (e.g. Bjorck et al,. 2018, Santurkar et al., 2018), batch normalisation tends to accelerate convergence during training. A dropout layer is used to prevent neural networks from overfitting the training data and thus generalizing poorly. The basic idea is to randomly drop units from the network. This prevents co-adaptation (different units of the network adapting similar weights) and provides regularization (lower the generalization error) (Srivastava et al., 2014).

Using these building blocks, we construct two types of convolutional neural networks in this study. The first type consists of several convolutional and max pooling layers, which downsample an image, followed by a couple of dense layers, which classify it. These models are most commonly employed to predict the label of an image, e.g. whether a certain object is in the image or not. The second type (U-Net) consists of two paths: one for down and one for upsampling (e.g. Long et al., 2015, Ronneberger et al., 2015). Each path typically consists of convolutional, max pooling, batch normalization and dropout layers as well as so-called skip connections, i.e. extra connections between nodes in different layers of a network that skip one or more layers (Orhan and Pitkov, 2018). In contrast to the first type, these models predict the label of each pixel of an image, allowing a much faster pixel by pixel prediction of images. We apply both of these types of models to compare their accuracy and speed as well as highlight advantages and disadvantages during training and application.

We can think of the training of these models as an optimization, which aims to minimize the difference between the predicted and the actual labels. This difference is calculated using a loss function 
(e.g. mean squared error, cross-entropy). Optimizers (e.g. stochastic gradient descent, adaptive moment estimation) allow us to minimize the loss function by adjusting the model weights via backpropagation (Rumelhart et al., 1986). We can monitor the optimization by tracking several metrics (e.g. loss, accuracy) during training. These learning curves are useful for hyperparameter tuning and to decide when training has been completed. Once training is complete, we can determine the performance of the model on the test set and if sufficient apply it to our entire data set.

On the technical side, we developed, trained and applied our models using a series of scripts implemented in Python 3, which we based upon several existing packages such as Segyio, NumPy (Van der Walt et al., 2011), Matplotlib (Hunter, 2007), Tensorflow (Abadi et al., 2016) and Keras (Chollet, 2015). Supervised machine learning workflows for seismic interpretation typically consist of six components: (1) load data and labels, (2) prepare data, (3) set up model, (4) train model, (5) tune model, and (6) apply model (e.g. Wrona et al, 2018). We describe these components in more detail before we employ our approach to specific examples.

First, we load seismic sections or volumes into $\underline{\text { NumPy }}$ arrays using the package Segyio. We label training data with a graphics editor (e.g. Inkscape or Adobe Illustrator) for the fault and salt interpretation or with a seismic interpretation software (e.g. Petrel or Kingdom) for the horizon interpretation. We load labelled sections saved as images using Matplotlib. Second, we standardize our seismic data (mean=0,

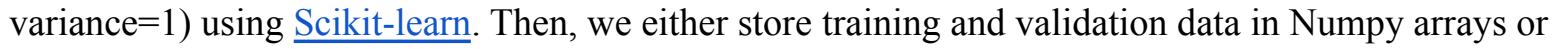
stream the data using data generators from Keras. Third, we set up our models using Keras, a high-level neural networks API built on top of Tensorflow. We use tensorflow-gpu to accelerate training. We initialize model weights with Keras' default settings (e.g. kernel initializer is glorot uniform). Fourth, we train our models using one of these loss functions: 
Mean squared error: $\quad M S E=\frac{1}{n} \sum_{i=1}^{n}\left(Y_{i}-\widehat{Y}_{i}\right)^{2}$

Binary cross-entropy: $\quad B C E=\frac{1}{n} \sum_{i=1}^{n} Y * \log \left(\widehat{Y}_{i}\right)+(1-Y) * \log \left(1-\widehat{Y}_{i}\right)$

Custom loss: $C L=F P / P+F N / N$

where $\mathrm{n}$ is the number of samples, $Y$ are the true labels and $\widehat{Y}$ are the predicted labels (as well as an

adaptive learning rate optimizer), FP are the false positives, $\mathrm{P}$ all positives, $\mathrm{FN}$ are the false negatives and $\mathrm{N}$ are all negatives. Fifth, we manually tune hyperparameters based on these metrics:

$\begin{array}{ll}\text { Binary accuracy: } & A C C=\frac{T P+T N}{F P+F N} \\ \text { Precision: } & \text { Pre }=\frac{T P}{T P+F P} \\ \text { Recall: } & R e c=\frac{T P}{T P+F N} \\ \text { F1-score: } & F 1=2 * \frac{P r e^{*} R e c}{P r e+\operatorname{Rec}} \\ \text { IOU score: } & I O U=\frac{T P}{T P+F P+F N}\end{array}$

where TP - true positives, TN - true negatives, FP - false positives, and FN false negatives. Sixth, we apply our models to entire data sets by iterating over points or windows. Our code, tutorials and examples are freely available at: https://github.com/thilowrona/seismic deep learning.

\section{FAULTS}

Our first example focuses on mapping faults, i.e. structural discontinuities that form when the crust is experiencing brittle deformation (e.g. during continental rifting of the northern North Sea). First, we approach this task as a binary classification problem, where we train our models to predict if there is a 
fault at each point of the section or not. We generate our training examples by manually labelling all faults visible in ten 2 -D seismic sections $(2801 \times 8096$ samples, $180 \times 20 \mathrm{~km})$ of the northern North Sea (Fig. 2A, B). Next, we extract squares $(128 \times 128$ samples $)$ from eight of these sections until we have 40,000 examples with a fault at the centre (label: 1) and 40,000 without (label: 0). Balancing these examples across classes makes it easier to train models. Next, we extract 10,000 examples from each of the remaining two seismic sections for validation (10\%) and testing $(10 \%)$. We use the training set to fit our model; a 2-D convolutional neural network consisting of three 2-D convolutional layers followed by max pooling and two dense layers. In total, the model has $8,413,442$ trainable parameters. We train the model over 10 epochs (one epoch refers one cycle through the entire training set) using 2500 batches consisting of 32 labelled training examples each (one batch refers to one set of training examples to work through before updating model weights). We use a binary cross-entropy loss function, an adaptive learning rate optimizer and a softmax activation for two classes. The training takes 16 minutes on our desktop machine with GPU support (see Appendix for specifications). 

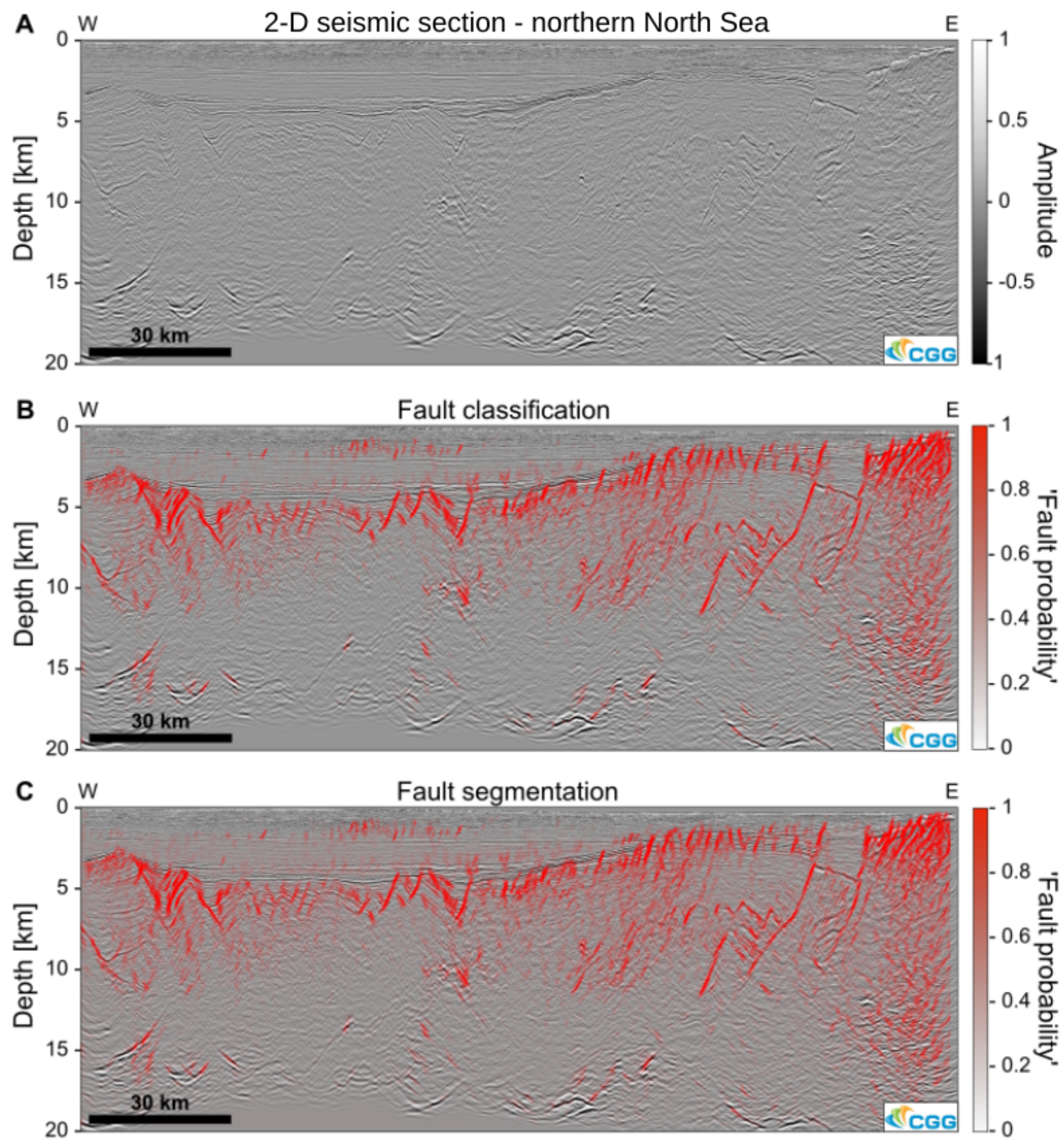

Figure 2. Automated fault interpretation in 2-D seismic section with 2-D convolutional neural networks. A) Seismic section $(180 \times 20 \mathrm{~km})$ of the northern North Sea rift. B) Seismic section with 'fault probability' (not true probability) prediction from 2-D convolutional neural network used for binary classification (duration of prediction: 55 minutes). C) Seismic section with fault segments predicted by U-Net type 2-D convolutional neural network used for semantic segmentation (duration of prediction: 5 seconds). Seismic data courtesy of CGG. 

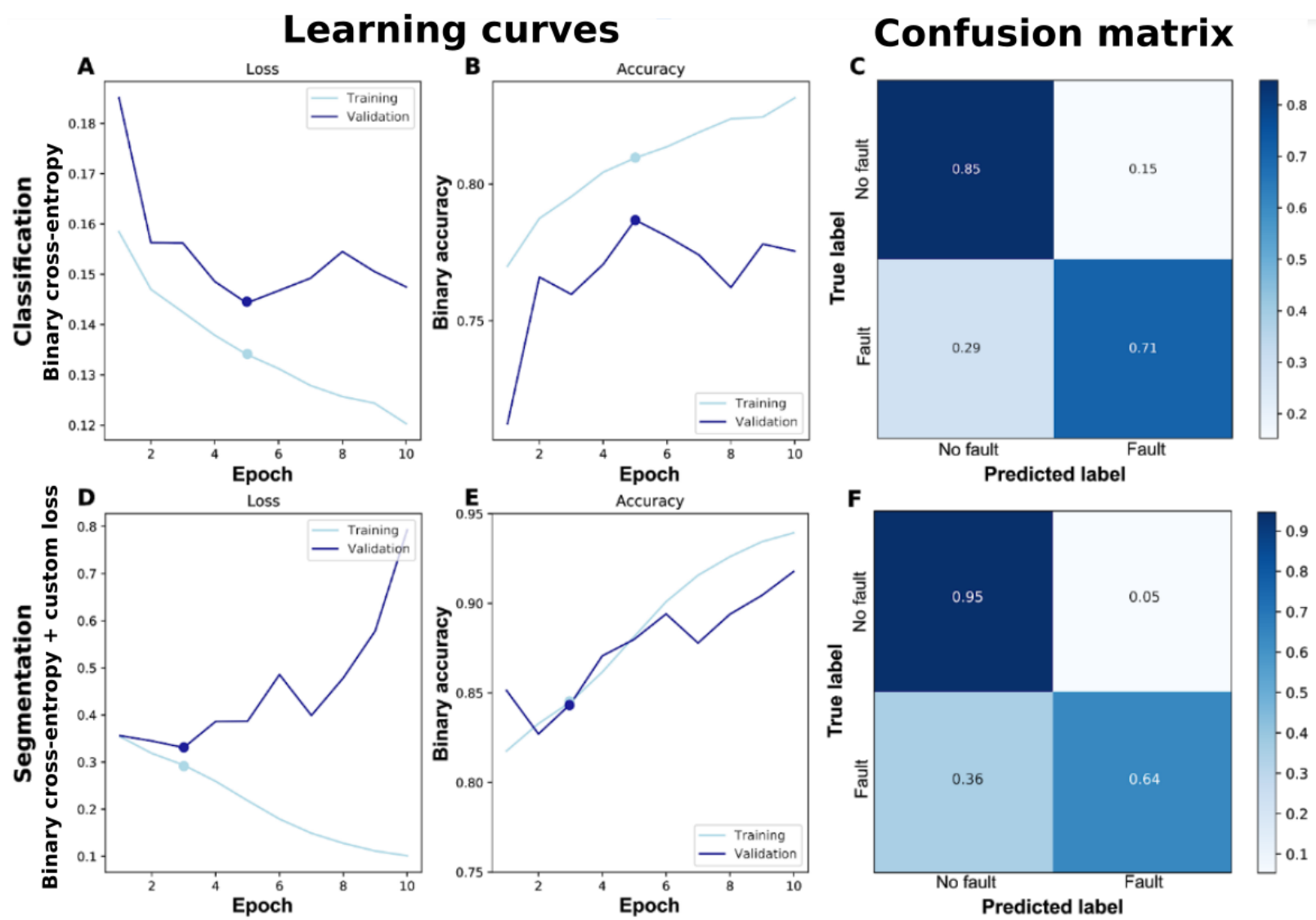

Figure 3. Learning curves and confusion matrices showing training results of classification ( $A, B$, and $C)$ and segmentation models (D, E, and F) for fault interpretation. A decrease in loss and increase in accuracy with time (epochs) indicates training progress. Dots indicate the final model (i.e. where validation loss was minimal). The confusion matrix cross-plots predicted and true labels, so that high values on the diagonal indicate high accuracy for the classes. Note that the relatively large fraction of false positives is somewhat by design, as we would rather find more faults than missing some (e.g. for $\mathrm{CO}_{2}$ storage).

During training, the loss (binary cross-entropy) decreases from 0.16 to 0.12 on the training set and from 0.19 to 0.14 on the validation set (Fig. $3 \mathrm{~A}$ ), while the binary accuracy increases from 0.77 to 0.83 on the training and from 0.71 to 0.79 on the validation set (Fig. 3B). We save the model after epoch 5, when the accuracy is highest and the loss lowest on the validation set.

The final model predicts $85 \%$ true positives (fault), $15 \%$ false positives, $71 \%$ true negatives (no fault) and 29\% false negatives (Fig. 3C). The model reaches a precision of 0.79 , a recall of 0.79 , a F1-score of 0.75 with a support of 10000 on the test set (Tab. 1). Precision describes the ability of a model to predict classes correctly, recall (or sensitivity) describes the ability of a model to find all 
examples of a class, F1 score is an equally weighted harmonic mean of precision and recall, and support is the number of examples tested. Since these values are relatively high, it makes sense to apply the model to an unseen seismic section.

\begin{tabular}{|c|c|c|c|c|c|c|}
\hline Model & Target & Method & Precision & Recall & F1-score & Support \\
\hline Model 1A & Faults & Classification & 0.79 & 0.79 & 0.75 & 10,000 \\
\hline Model 1B & Faults & Segmentation & 0.83 & 0.82 & 0.76 & 10,000 \\
\hline Model 2 & Salt bodies & Classification & 0.91 & 0.91 & 0.91 & 10,000 \\
\hline Model 3 & Horizon & Classification & 0.92 & 0.91 & 0.88 & 10,000 \\
\hline
\end{tabular}

Table 1. Model metrics for fault interpretation calculated on a test set at the end of training.

Applying the model to an unseen 2-D seismic section of the northern North Sea takes 120

minutes on our machine, because the model has to predict the label at the centre of each of the $21,983,584$ pixels of the section (no smoothing). Overall, the model predicts all major faults in the upper crust, but also labels some steeply-inclined stratigraphic reflections as faults (Fig. 2A,B). The 'true' faults show typical geometries including: (1) plane, (2) listric, and (3) kinked surfaces (Fig. 2C) (Bell et al., 2014). The model is also able to predict fault intersections, such as criss-crossing and splaying faults as well as associated structures, such as en échelon faults and rotated fault blocks.

Next, we approach this task as a semantic segmentation problem, where we train our model to predict a pixel-wise label of the image. Pixel values of 1 indicate a fault and 0 no fault. We can again use our seismic section and the labelled interpretation from the northern North Sea to extract 80000 squares $(128 \times 128$ samples $)$ together with the corresponding labels $(128 \times 128$ samples $)$, respectively. Next, we split these pairwise examples into training (80\%), validation $(10 \%)$ and test set $(10 \%)$. We use the training set to fit an U-Net; a 2-D convolutional neural network consisting of a contracting and an expanding path. These models excel at segmentation tasks, as they combine pixel-wise classification and localisation. Our U-Net is symmetric with each side consisting of thirteen convolutional layers each followed by a batch 
normalisation, max pooling and dropout layer. In total, the model has 31,042,369 trainable parameters. We train the model over 10 epochs using 2500 batches consisting of 32 labeled training examples each. We use a binary cross-entropy combined with a custom loss function (normalized false positives + normalized false negatives) and an adaptive learning rate optimizer. We chose this loss function, because it minimizes both false negative and false positive predictions for imbalanced classes (e.g. fault vs no fault). The training takes 2 hours 16 minutes on our desktop machine with GPU support (see Appendix for specifications).

During training, the loss decreases from 0.35 to 0.29 on the training set and from 0.36 to 0.33 on the validation set (Fig. 3D), while the binary accuracy increases from 0.82 to 0.84 on the training and from 0.82 to 0.84 on the validation set (Fig. 3B). We save the model after epoch 3, when the loss is lowest on the validation set.

The final model predicts $64 \%$ true positives (fault), $36 \%$ false positives, $95 \%$ true negatives (no fault) and 5\% false negatives (Fig. 3C). The model reaches a precision of 0.83 , a recall of 0.82 , a F1-score of 0.76 with a support of 10,000 on the test set (Tab. 1). All these values are reasonably high, so it makes sense to apply the model to the entire seismic section.

Applying the model to an unseen 2-D seismic section of the northern North Sea takes only 5 seconds on our machine, because the model simultaneously predicts $128 \times 128$ of the $21,983,584$ pixels for each batch of the section (no smoothing or overlap). The model is thus able to predict the faults just as well as the previous model, but in a fraction of the time.

\section{SALT BODIES}

Our second example focuses on mapping salt bodies in three dimensions. Given that salt forms complex bodies, which are difficult to label in 3-D as segmentation masks, we approach this task as a 
binary classification, where we train a 3-D convolutional neural network using 3-D seismic reflection data. We label salt on 5 seismic inlines and 5 crosslines to generate a total of 1,000,000 examples in the form of small cubes $(16 \times 16 \times 16$ samples $)$. These examples are already relatively balanced across the two classes (salt, no salt) in this dataset. Next, we split these examples into exclusive training (8 lines, $80 \%$ ), validation (1 line, 10\%) and test set (1 line, 10\%). The training set is used to fit our model: a 3-D U-Net consisting of 183-D convolutional, three 3-D max pooling and three upsampling layers . In total, the model has 5,884,033 trainable parameters. We train the model over 10 epochs using 25,000 batches consisting of 32 labelled training examples each. We use a binary cross entropy loss function and an adaptive learning rate optimizer. The loss function is masked to allow training of a 3-D model with 2-D labels (sensu Tschannen et al., 2020). The training took about 35 minutes on our desktop machine with GPU support (see Appendix for specifications).
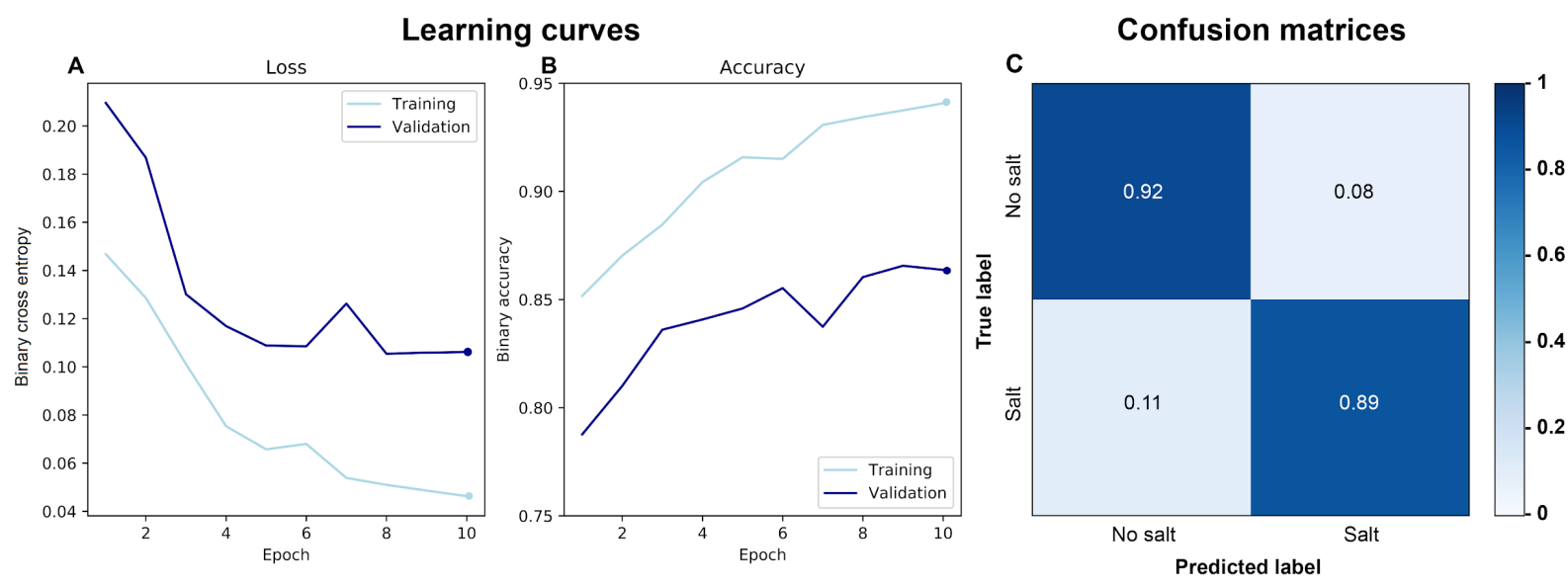

Figure 4. Learning curves and confusion matrices showing training results of classification model for salt interpretation. $A$ decrease in loss $(A)$ and increase in accuracy $(B)$ with time (epochs) indicates training progress. The final model is the one with the lowest validation loss (i.e. after 10 epochs). The confusion matrix cross-plots predicted and true labels, so that high values on the diagonal indicate high accuracy for the classes.

During training, the loss (binary cross entropy) decreases from 0.15 to 0.05 on the training set and from 0.21 to 0.11 on the validation set, and the binary accuracy increases from 0.85 to 0.94 on the training 
set and from 0.79 to 0.85 on the validation set (Fig. 4A,B). Constant losses and accuracies with time indicate that training has been completed after 10 epochs.

The final model predicts $89 \%$ true positives (salt), 11\% false positives, $92 \%$ true negatives (no salt) and $8 \%$ false negatives (Fig. 4C). The model achieves a precision of 0.91 , a recall of 0.91 , a F1-score of 0.91 with a support of 10,000 on the test set (Tab. 1). These values give us confidence that we can apply the model.

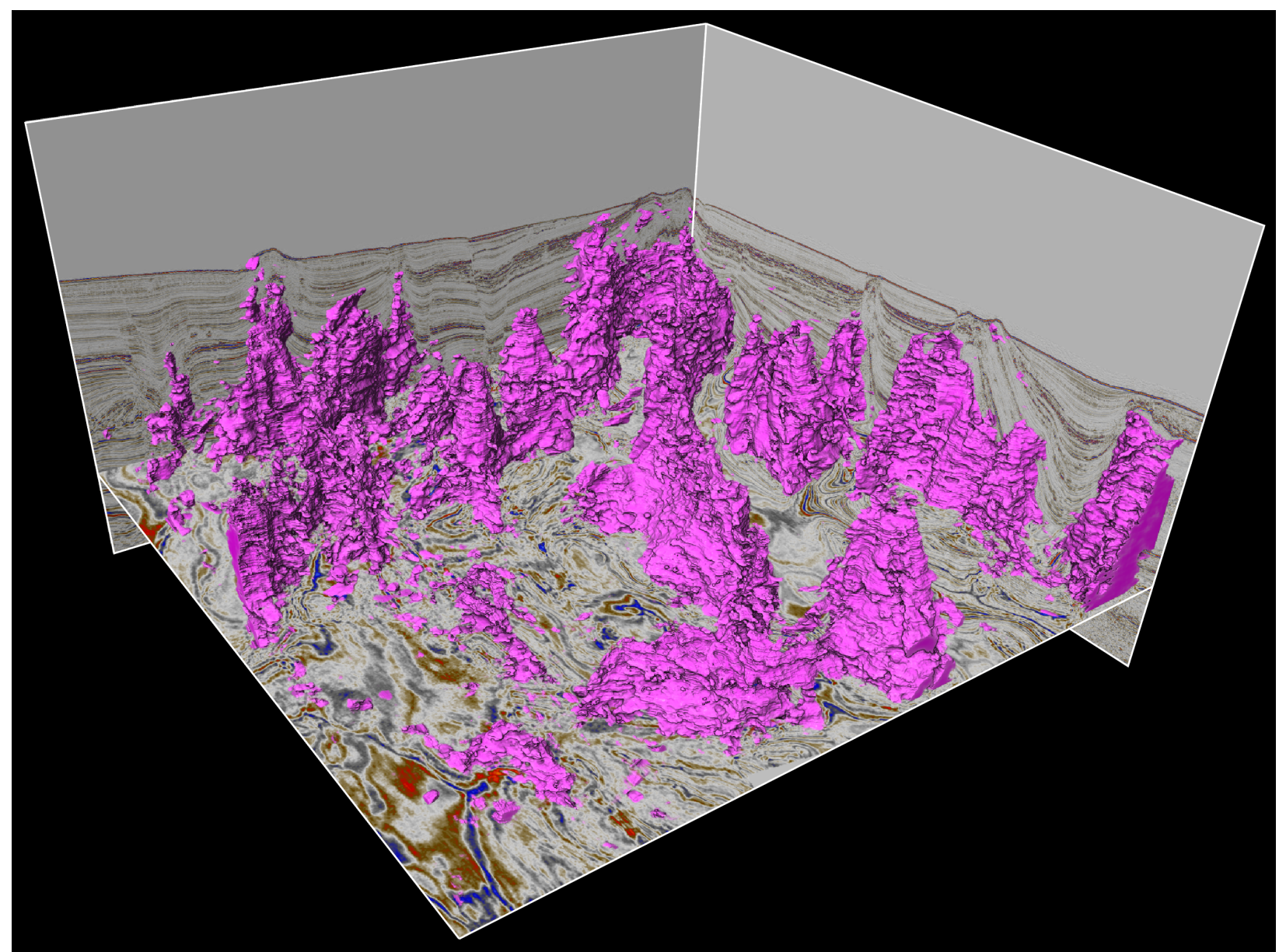

Figure 5: Salt diapirs mapped in 3-D seismic reflection data with a 3-D deep convolutional neural network. This volume has an approximate size of $60 \times 60 \times 8 \mathrm{~km}$.

Applying the model to the entire 3-D seismic volume takes 49 minutes on our machine. Overall, the model is able to predict a number of typical 3-D structures, such as salt walls and diapirs (Fig. 5). 


\section{HORIZONS}

Our third example focuses on mapping a seismic horizon in 3-D seismic reflection data from the northern North Sea. We approach this problem as a binary segmentation, using a 3-D U-Net. To generate examples, we map a horizon in one area of the dataset using a seismic interpretation software. This provides us with 100,000 examples in the form of small cubes $(32 \times 32 \times 32$ samples $)$. Next, we sort these examples into exclusive sets: one for training $(80 \%)$ and one for validation $(10 \%)$ and one for testing $(10 \%)$. We use the training set to fit our model: a 3-D U-Net consisting of 183-D convolutional, batch three 3-D max pooling and three upsampling layers. In total, the model has 5,884,033 trainable parameters. We train our model over 10 epochs using 2500 batches consisting of 32 labelled training examples each. We use a masked binary cross entropy loss function (sensu Tschannen et al., 2020) and an adaptive learning rate optimizer. The training took about 38minutes on our desktop machine with GPU support (see Appendix for specifications).
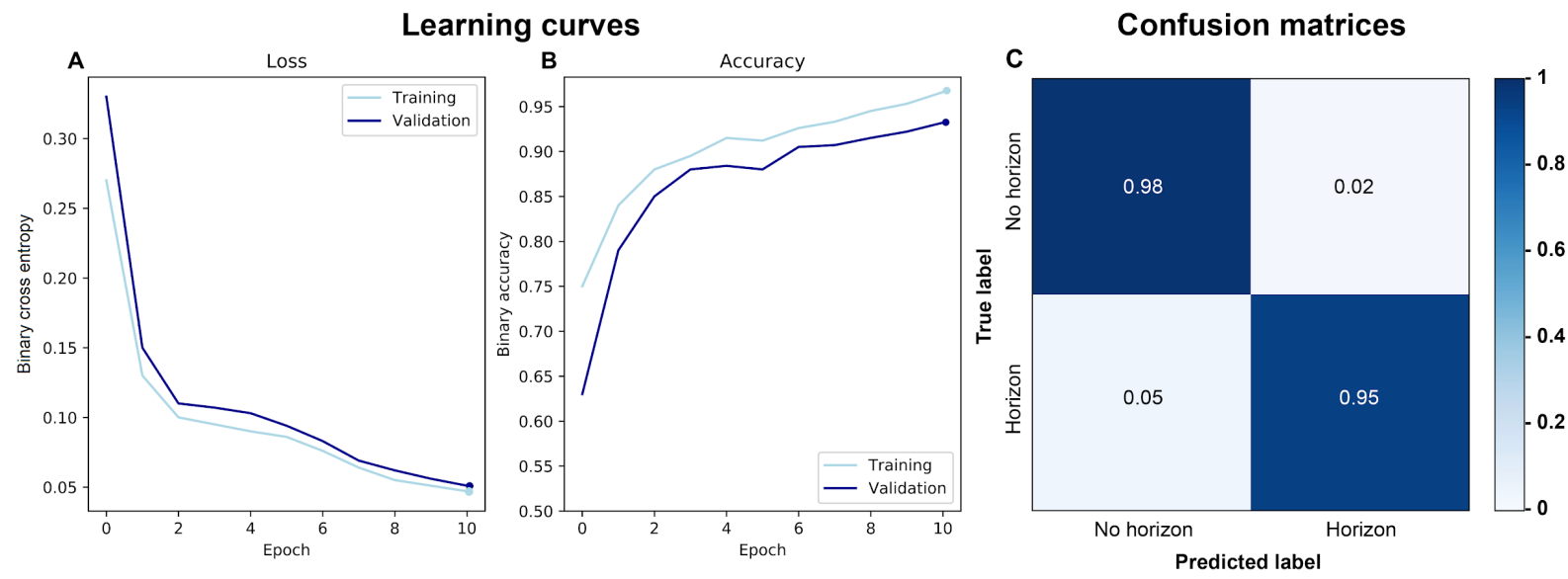

Figure 6. Learning curves and confusion matrices showing training results of classification model for horizon interpretation. A decrease in loss (A) and increase in accuracy (B) with time (epochs) indicates training progress. The final model is the one with the lowest validation loss (i.e. after 10 epochs). The confusion matrix cross-plots predicted and true labels, so that high values on the diagonal indicate high accuracies for the classes. 
During training, the loss (binary cross-entropy) decreases from 0.27 to 0.047 on the training set and from 0.33 to 0.051 on the validation set (Fig. 6A). At the same time, the binary accuracy increases from 0.75 to 0.966 on the training and from 0.63 to 0.932 on the validation set (Fig. 6B). Plateauing of loss and accuracy with time suggests that training has been completed.

The final model predicts $95 \%$ true positives (horizon), $5 \%$ false positives, $98 \%$ true negatives (no horizon) and $2 \%$ false negatives (Fig. 6C). The model reaches a precision of 0.92 , a recall of 0.91 , a F1-score of 0.88 with a support of 10,00 on the test set (Tab. 1). Given that these values are sufficiently high, we can apply the model to a neighbouring area.

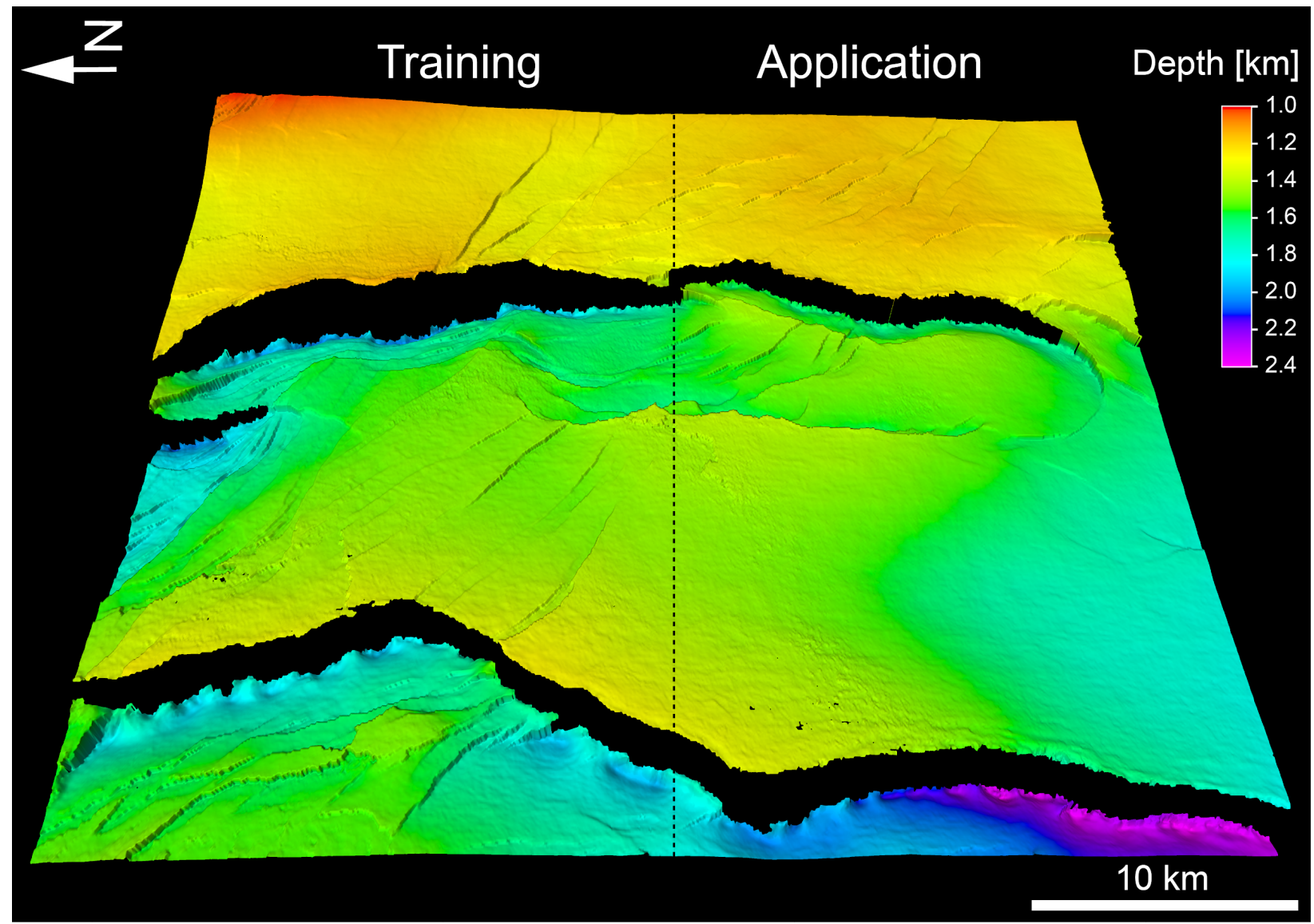

Figure 7: Geological horizon mapped in 3-D seismic reflection data using a deep 3-D convolutional neural network. 
Applying the model to the right hand side of Figure 7 takes 1 hours 12 minutes. A direct visual comparison between the training data and the predictions shows that the model is able to map a continuous horizon with the same geological structures (large and small normal faults) on unseen data.

\section{DISCUSSION}

Our models highlight that we can use deep learning for typical seismic interpretation tasks, such as fault, salt and horizon mapping in two and three dimensions (Figs. 2, 5, 7). Using labelled training data, we can train a set of 2-D and 3-D convolutional neural networks to perform these tasks in as little as 5 seconds. This allows us to map 2-D and 3-D geological structures (e.g. faults, geobodies and horizons) in great detail, over vast areas, and at high accuracy. This has far-reaching implications. For instance, if we are able to map entire rifts and rift systems in 3-D seismic reflection data, we could compare the architecture of these systems to 3-D geodynamic models, which, in turn, helps us understand the rheology of the lithosphere and the spatial evolution of fault rift systems. Mapping every stratigraphic horizon in these systems would help us reconstruct their temporal evolution in much greater detail than previously possible. While this example focuses on tectonic faults in continental rifts, a similar approach is feasible in different disciplines working with 2-D and 3-D seismic reflection data, whether it concerns the study of submarine channels, magmatic intrusions or salt tectonics.

In the following, we discuss: (1) the potential of deep learning in geophysics, (2) how generalisable these methods are to other problems in geophysics, and (3) what are the challenges and few remedial strategies. We think that there are three main reasons regarding the suitability of deep learning techniques for analysis of 3-D geophysical data. First, geophysical datasets are large (GBs to TBs); a key requirement for the implicit feature extraction of deep learning (Chen \& Lin, 2014). Second, we already have large volumes of historical training data labelled by experts and if necessary, we can label even more 
training data. Third, the translation invariance of convolutional neural networks has made deep learning very effective in analyzing images; a property we too can exploit in geophysics.

With deep learning transforming the way we analyze data, we can ask ourselves how transferable these methods are to other problems in geophysics. First, it is worth noting that deep learning does not require feature engineering and is thus not limited to a specific type of geophysical data. Appropriate transformations (e.g. scaling) can however greatly improve model performance. Second, the models presented here are still relatively simple in terms of architecture, so that users can easily tweak them to adapt the workflow to their problem. Moreover, it is possible to optimize the hyperparameters of these models to further increase accuracy and speed. To encourage these developments, we made our code freely available on: https://github.com/thilowrona/seismic deep learning. Third, it is possible to apply our workflow to map a variety of geological structures (e.g. volcanoes, channels or craters) in different types of geophysical data (radar, magnetic, gravity). Finally, we can explore applications of deep learning in other tasks in seismic interpretation. Uncertainty quantification, for example, is extremely difficult in manual seismic interpretations. While our models only predict a proxy for the probability of detecting a certain geological structure (e.g. Fig. 2B, C), we can envision incorporating uncertainty in the training data and architecture of these models (e.g. Mosser et al., 2020; Feng et al., 2021). Accurate predictions of subsurface rock properties with uncertainty are extremely important to risk evaluations of $\mathrm{CO}_{2}$ storage sites and geothermal reservoirs. Bayesian techniques like Variational Inference for deep neural networks can be used to approximate the posterior predictive distribution. These are optimisation based techniques that are much faster and tractable than full Markov Chain Monte Carlo methods, although they are known to under-estimate the uncertainty bounds (e.g. Blei et al., 2017).

While these techniques are very powerful, it is also worth highlighting some of the challenges (and potential remedies) associated with deep learning. For example, pixel-wise predictions are more 
time-consuming with classification instead of segmentation models (e.g. Zhao, 2018). Labelling training data for segmentation models, in particular in 3-D, is challenging and time-consuming, too. A masked loss function allows training with 3-D models and data using 1-D- and 2-D labels (see Tschannen et al., 2020 for more details). This function restricts the loss calculation during training using a mask, which is one where a label is available and zero where it is absent.

Another challenge is determining the optimal number of training examples. While we found that around 100,000 examples provide a good trade-off between prediction accuracy and training time, this point is worth investigating further. When labels are only available for a small subset, it is possible to increase the size of the training set using data augmentation techniques. When there are no labels available, we (as a user or community) have to spend time labelling data manually. Active learning, where models can query users to label certain data points, is a promising approach worth exploring in seismic interpretation. Moreover, we can explore unsupervised learning methods (e.g. autoencoders), which do not require labels.

Another issue of deep learning is overfitting. Deep neural networks can provide accurate predictions on the training set, but fail to generalize on new data (i.e. overfitting) (Domingos, 2012). To identify overfitting, we can compare model predictions between the training and validation set. Moreover, it is advisable to start with a simple model and successively increase complexity as necessary. Furthermore, regularization techniques, such as dropout (Srivastava et al., 2014), can help us limit overfitting.

At last, it is worth mentioning that neural networks are still, to a large degree, a black box, offering only sporadic insights into their inner workings. So while these models can perform many tasks at high accuracy and speed, we are just beginning to understand which features these models use and how they make decisions, so interpretability remains a key focus area of machine learning research (e.g. Ribeiro et al., 2016; Lundberg and Lee, 2017; Molnar, 2018). 


\section{CONCLUSIONS}

Here, we demonstrate how to use deep learning to analyse geophysical data sets at high accuracy and limited time. We show that deep convolutional neural networks are able to perform key tasks, such as (1) mapping tectonic faults, (2) extracting geobodies, and (3) tracing stratigraphic horizons. As such, this study highlights new ways to efficiently analyze large volumes of geophysical data using freely available methods. To summarize, this study shows that deep learning has the potential to drastically increase the speed and accuracy of geophysical data analyses.

\section{ACKNOWLEDGEMENTS}

We would like to thank the journal editors (Yongyi Li, John T. Etgen and Erika Gasperikova) and the reviewers (Rocky Roden, Xinming $\mathrm{Wu}$, two anonymous) for their extremely constructive and helpful comments, which significantly improved the manuscript. Furthermore, we thank The Norwegian Academy of Science and Letters (VISTA), The University of Bergen and The Initiative and Networking Fund of the Helmholtz Association through the project "Advanced Earth System Modelling Capacity (ESM) and The Geo.X Network for supporting this work. S. Brune has been funded through the Helmholtz Young Investigators Group CRYSTALS (VH-NG-1132). We are grateful to CGG, in particular Stein Åsheim, for the permission to present this data and publish this work. We thank Schlumberger for providing the software Petrel 2019® and Leo Zijerveld for IT support.

\section{REFERENCES}

Abadi, M., Barham, P., Chen, J., Chen, Z., Davis, A., Dean, J., et al. (2016). TensorFlow: A system for large-scale machine learning. In In12th Symposium on Operating Systems Design and Implementation (pp. 265-283). Retrieved from http://arxiv.org/abs/1605.08695 
Araya-Polo, M., Dahlke, T., Frogner, C., Zhang, C., Poggio, T., \& Hohl, D. (2017). Automated fault detection without seismic processing. The Leading Edge, 36(3), 208-214.

https://doi.org/10.1190/tle36030208.1

Bell, R. E., Jackson, C. A. L., Whipp, P. S., \& Clements, B. (2014). Strain migration during multiphase extension: Observations from the northern North Sea. Tectonics, 33(10), 1936-1963.

https://doi.org/10.1002/2014tc003551

Bjorck, J., Gomes, C., Selman, B., \& Weinberger, K. Q. (2018). Understanding batch normalization. In Advances in Neural Information Processing Systems.

Blei, D. M., Kucukelbir, A., \& McAuliffe, J. D. (2017). Variational Inference: A Review for Statisticians. Journal of the American Statistical Association. https://doi.org/10.1080/01621459.2017.1285773

Bond, C. E., Lunn, R. J., Shipton, Z. K., \& Lunn, A. D. (2012). What makes an expert effective at interpreting seismic images? Geology, 40(1), 75-78. https://doi.org/10.1130/g32375.1

Butler, K. T., Davies, D. W., Cartwright, H., Isayev, O., \& Walsh, A. (2018). Machine learning for molecular and materials science. Nature. https://doi.org/10.1038/s41586-018-0337-2

Carleo, G., \& Troyer, M. (2017). Solving the quantum many-body problem with artificial neural networks. Science, 355(6325), 602-606. https://doi.org/10.1126/science.aag2302

Cartwright, J., \& Huuse, M. (2005). 3D seismic technology: the geological 'Hubble.' Basin Research, 17(1), 1-20. https://doi.org/10.1111/j.1365-2117.2005.00252.x

Chen, X. W., \& Lin, X. (2014). Big data deep learning: Challenges and perspectives. IEEE Access. https://doi.org/10.1109/ACCESS.2014.2325029

Chollet, F. (2015). Keras: The Python Deep Learning library. Keras.Io.

Coléou, T., Poupon, M., \& Azbel, K. (2003). Unsupervised seismic facies classification: A review and comparison of techniques and implementation. The Leading Edge, 22(10), 942-953.

https://doi.org/10.1190/1.1623635

Domingos, P. (2012). A few useful things to know about machine learning. Communications of the ACM. https://doi.org/10.1145/2347736.2347755

Esteva, A., Kuprel, B., Novoa, R. A., Ko, J., Swetter, S. M., Blau, H. M., \& Thrun, S. (2017).

Dermatologist-level classification of skin cancer with deep neural networks. Nature, 542(7639), 115.

Retrieved from https://www.nature.com/articles/nature21056.pdf

Fent, R., Grana, D. and Bailing N. (2021). Uncertainty quantification in fault detection using convolutional neural networks. GEOPHYSICS 0: 1-45. https://doi.org/10.1190/geo2020-0424.1

Gramstad, O., Nickel, M., Goledowski, B., \& Etchebes, M. (2020). Strategies in picking training data for 3D convolutional neural networks in stratigraphic interpretation. SEG Technical Program Expanded Abstracts : 1641-1645. https://doi.org/10.1190/segam2020-3427602.1 
Guillen, P., Larrazabal*, G., González, G., Boumber, D., \& Vilalta, R. (2015). Supervised learning to detect salt body. In SEG Technical Program Expanded Abstracts 2015 (pp. 1826-1829). Society of Exploration Geophysicists. https://doi.org/10.1190/segam2015-5931401.1

Hunter, J. D. (2007). Matplotlib: A 2D graphics environment. Computing in Science and Engineering. https://doi.org/10.1109/MCSE.2007.55

LeCun, Y., Bengio, Y., \& Hinton, G. (2015). Deep learning. Nature, 521(7553), 436-444. https://doi.org/10.1038/nature14539

Li, W. (2018). Classifying geological structure elements from seismic images using deep learning. SEG Technical Program Expanded Abstracts : 4643-4648. https://doi.org/10.1190/segam2018-2998036.1

Liu, Z., Cao, J., Lu, Y., Chen, S., \& Liu, J. (2019). A seismic facies classification method based on the convolutional neural network and the probabilistic framework for seismic attributes and spatial classification. Interpretation. https://doi.org/10.1190/int-2018-0238.1

Long, J., Shelhamer, E., \& Darrell, T. (2015). Fully convolutional networks for semantic segmentation. In Proceedings of the IEEE Computer Society Conference on Computer Vision and Pattern Recognition. https://doi.org/10.1109/CVPR.2015.7298965

Lundberg, S. M., \& Lee, S. I. (2017). A unified approach to interpreting model predictions. In Advances in Neural Information Processing Systems.

de Matos, M. C., Osorio, P. L., \& Johann, P. R. (2007). Unsupervised seismic facies analysis using wavelet transform and self-organizing maps. Geophysics, 72(1), P9-P21.

https://doi.org/10.1190/1.2392789

Molnar, C. (2018). Interpretable Machine Learning.

Https://Christophm.Github.Io/Interpretable-Ml-Book/. https://doi.org/10.1007/978-1-4419-0790-5

Mosser, L., Purves S. and Naeini E.Z. (2020). Deep Bayesian Neural Networks for Fault Identification and Uncertainty Quantification. Conference Proceedings, First EAGE Digitalization Conference and Exhibition, Nov 2020, Volume 2020, p.1 - 5. https://doi.org/10.3997/2214-4609.202032036

Nair, V., \& Hinton, G. E. (2010). Rectified linear units improve Restricted Boltzmann machines. In ICML 2010 - Proceedings, 27th International Conference on Machine Learning.

Orhan, A. E., \& Pitkow, X. (2018). Skip connections eliminate singularities. In 6th International Conference on Learning Representations, ICLR 2018 - Conference Track Proceedings.

Peters, B., Haber, E., \& Granek, J. (2019). Neural networks for geophysicists and their application to seismic data interpretation. Leading Edge. https://doi.org/10.1190/tle38070534.1

Qian, F., Yin, M., Liu, X.-Y., Wang, Y.-J., Lu, C., \& Hu, G.-M. (2018). Unsupervised seismic facies analysis via deep convolutional autoencoders. Geophysics, 83(3), A39-A43.

https://doi.org/10.1190/geo2017-0524.1 
Ribeiro, M. T., Singh, S., \& Guestrin, C. (2016). "Why should i trust you?" Explaining the predictions of any classifier. In Proceedings of the ACM SIGKDD International Conference on Knowledge Discovery and Data Mining. https://doi.org/10.1145/2939672.2939778

Ronneberger, O., Fischer, P., \& Brox, T. (2015). U-net: Convolutional networks for biomedical image segmentation. In Lecture Notes in Computer Science (including subseries Lecture Notes in Artificial Intelligence and Lecture Notes in Bioinformatics). https://doi.org/10.1007/978-3-319-24574-4_28

Rumelhart, D. E., Hinton, G. E., \& Williams, R. J. (1986). Learning representations by back-propagating errors. Nature. https://doi.org/10.1038/323533a0

Santurkar, S., Tsipras, D., Ilyas, A., \& Madry, A. (2018). How does batch normalization help optimization? In Advances in Neural Information Processing Systems.

Srivastava, N., Hinton, G., Krizhevsky, A., Sutskever, I., \& Salakhutdinov, R. (2014). Dropout: A simple way to prevent neural networks from overfitting. Journal of Machine Learning Research.

Tschannen, V., Delescluse, M., Ettrich, N., \& Keuper, J. (2020). Extracting horizon surfaces from 3D seismic data using deep learning. GEOPHYSICS. https://doi.org/10.1190/geo2019-0569.1

Waldeland, A. U., Jensen, A. C., Gelius, L.-J., \& Solberg, A. H. S. (2018). Convolutional neural networks for automated seismic interpretation. The Leading Edge, 37(7), 529-537. Retrieved from https://library.seg.org/doi/pdf/10.1190/tle37070529.1

Van Der Walt, S., Colbert, S. C., \& Varoquaux, G. (2011). The NumPy array: A structure for efficient numerical computation. Computing in Science and Engineering. https://doi.org/10.1109/MCSE.2011.37

Wrona, T., Pan, I., Gawthorpe, R. L., \& Fossen, H. (2018). Seismic facies analysis using machine learning. Geophysics, 83(5), O83-O95. https://doi.org/10.1190/Geo2017-0595.1

Wu, X. M., \& Fomel, S. (2018). Automatic fault interpretation with optimal surface voting. Geophysics, 83(5), O67-O82. https://doi.org/10.1190/Geo2018-0115.1

Wu, X., Yunzhi Shi, L.L. \& Fomel, S. (2019), FaultSeg3D: Using synthetic data sets to train an end-to-end convolutional neural network for 3D seismic fault segmentation, GEOPHYSICS 84: IM35-IM45. https://doi.org/10.1190/geo2018-0646.1

Zhang, C., Frogner, C., Araya-Polo, M., \& Hohl, D. (2014). Machine-learning Based Automated Fault Detection in Seismic Traces. In 76th EAGE Conference and Exhibition 2014.

Zhao, T. (2019). Seismic facies classification using different deep convolutional neural networks. 2018 SEG International Exposition and Annual Meeting, SEG 2018, 2046-2050.

https://doi.org/10.1190/segam2018-2997085.1

Zhou, H., Xu, S., Ionescu, G., Laomana, M., \& Weber, N. (2020). Salt interpretation with U-SaltNet. SEG Technical Program Expanded Abstracts : 1434-1438. https://doi.org/10.1190/segam2020-3423283.1 


\section{SUPPLEMENTARY MATERIAL}

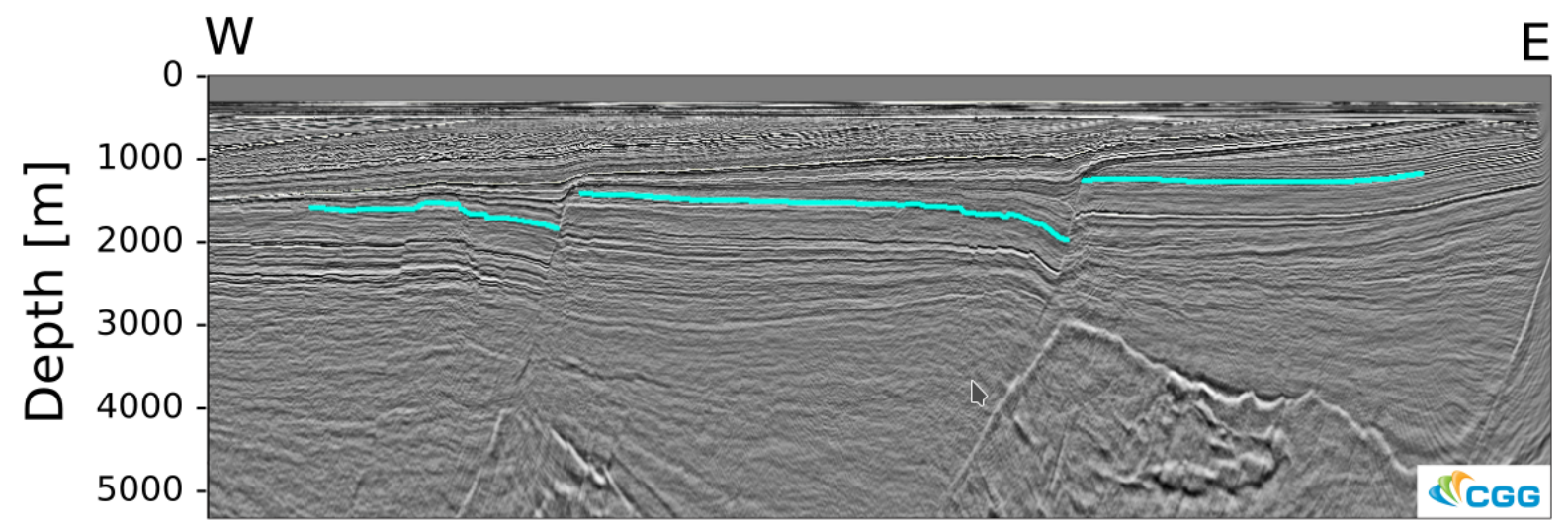

Supplementary Figure 1: 2-D seismic section with stratigraphic horizon predicted by deep convolutional neural network.

\section{Computer specifications}

System Information

Time of this report: 10/1/2019, 16:38:37

Operating System: Windows 10 Enterprise 64-bit (10.0, Build 16299) (16299.rs3_release_svc.180808-1748)

Language: Norwegian Bokmål (Regional Setting: Norwegian Bokmål)

System Manufacturer: HP

System Model: HP Z6 G4 Workstation

BIOS: Default System BIOS

Processor: Intel(R) Xeon(R) Gold 6128 CPU @ 3.40GHz (24 CPUs), 3.4GHz

Memory: 65536MB RAM

Available OS Memory: 65236MB RAM

Page File: $13022 \mathrm{MB}$ used, $76606 \mathrm{MB}$ available

Windows Dir: C: IWINDOWS

DirectX Version: DirectX 12

DX Setup Parameters: Not found

User DPI Setting: 96 DPI (100 percent)

System DPI Setting: 144 DPI (150 percent)

DWM DPI Scaling: UnKnown 
Miracast: Available, with HDCP

Microsoft Graphics Hybrid: Not Supported

DxDiag Version: 10.00.16299.0015 64bit Unicode

Display Devices

Card name: NVIDIA GeForce GTX $1080 \mathrm{Ti}$

Manufacturer: NVIDIA

Chip type: GeForce GTX $1080 \mathrm{Ti}$

AC type: Integrated RAMDAC

Device Type: Full Device

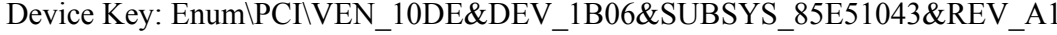

Device Status: 0180200A

[DN_DRIVER_LOADED|DN_STARTED|DN_DISABLEABLE|DN_NT_ENUMERATOR|DN_NT_DRIVER]

Device Problem Code: No Problem

Driver Problem Code: Unknown

Display Memory: 43744 MB

Dedicated Memory: $11127 \mathrm{MB}$

Shared Memory: 32617 MB

Current Mode: 1920 x 1080 (32 bit) $(32 \mathrm{~Hz})$ 